И. КЕНС

\title{
ОПТИМАЛЬНЫЙ СИНТЕЗ УПРАВЛЕНИЯ СИСТЕМЫ С ИНФОРМАТИВНЫМИ ПЕРЕМЕННЫМИ
}

\author{
(Представлена Н. Алумяэ)
}

\begin{abstract}
Рассматривается задача оптимального управления движением динамической системы с информативными переменными. Подынтегральная функция критерия принимается выпуклой по управлению, удовлетворяющему условию типа норм-ограничения. Дается класснфикация оптимальных режимов. Исходя из построенных экстремальных регуляторов выводится вид уравнения Беллмана и условий понижения размерности задачи. Результаты иллюстрируются на примере субоптимальной стабилизации движений псевдоавтономных систем.
\end{abstract}

1. Рассмотрим уравнения возмущений $x \equiv 0$ системы $\left[{ }^{1}\right]$

$$
\begin{gathered}
x=X(t, x)+M(t, x) u, X(t, 0) \equiv 0, \quad M=\left\|m_{v s}(q)\right\|, \\
u=\left(u_{s}\right)^{*} \in \omega(v=\overline{1, n,} s=\overline{1, r}), \\
X=\left(X_{v}(q)\right)^{*}, \quad q=\left(t, x_{v}\right)^{*} \in Q: t \geqslant 0, \quad|x|<\infty ; \\
1 \leqslant \operatorname{rank} M=r \leqslant n, \quad|x|^{2}=\sum_{v=1}^{n} x_{v}^{2}, \\
x=\left(x_{v}\right)^{*}=\left(x_{\alpha}^{\prime}, x^{\prime \prime}\right)^{*}, \quad x^{\prime}=\left(x_{\alpha}^{\prime}\right)^{*}, \quad x^{\prime \prime}=\left(x^{\prime \prime}\right)^{*} ; \\
X_{v}, m_{v s} \subset C_{1}(Q)(\alpha=\overline{1, l}, \beta=\overline{1, n-l}, l \leqslant n) .
\end{gathered}
$$

Допустимые управления - кусочно-непрерывные в $T=[0, \infty)$ векторфункции $u(t)$ со значениями в выпуклом компакте $\omega$

$$
0 \leqslant v(u) \leqslant v^{0}=\text { const, } \quad \omega_{0}=\omega \backslash 0, \quad v(u) \subset C\left(E^{r}\right),
$$

где

$$
\begin{gathered}
v\left(\alpha_{0} u\right)=\alpha_{0} v(u), \quad 0 \leqslant \alpha_{0} \in E^{1} ; \quad v \subset C_{2}\left(E_{0}^{r}\right)(v(u)=u \cdot \nabla v(u)), \\
v\left(u_{2}\right)-v\left(u_{1}\right)>\left(u_{2}-u_{1}\right) \cdot \nabla v\left(u_{1}\right), \quad\left|u_{2}\right|\left|u_{1}\right|>\left|\left(u_{2} \cdot u_{1}\right)\right|\left(E_{0}^{r}=E^{r} \backslash 0\right), \\
v(u)>v(0)=0, \quad u \neq 0, \quad \nabla v(u)=\left(\partial v / \partial u_{s}\right)^{*} \quad(s=\overline{1, r}) .
\end{gathered}
$$

Допустимыми будем считать управления, приводящие $x^{\prime}(t)$ на множество цели $x^{\prime}=0$. Здесь $t_{1}-$ момент примыкания: $x^{\prime}\left(t, x_{0}\right) \rightarrow 0$ при $t \rightarrow t_{1}-0$.

Введем обратимое преобразование $\xi_{v}=\xi_{v}(t, x)$ вида 


$$
\xi=\left(\xi_{v}\right)^{*}=\left(y^{\prime}{ }_{i}(q), y^{\prime \prime}{ }_{j}(q), \gamma_{\lambda}(q)\right)^{*}, \xi_{v}(t, 0) \equiv 0, y=\left(y_{m}\right)^{*}=\left(y^{\prime}{ }_{i}, y^{\prime \prime}{ }_{j}\right)^{*},
$$

$$
\gamma=\left(\gamma_{\lambda}\right)^{*}, y_{m}(q) \geqslant 0 ; \quad y_{m}, \gamma_{\lambda} \subset C(Q), 1 \leqslant \operatorname{dim} y=k \leqslant l=\operatorname{dim} x^{\prime},
$$

$P=\left\|p_{v \sigma}(q)\right\|, \quad p_{v \sigma}=\partial \xi_{v} / \partial x_{\sigma}, \quad \operatorname{det} P \neq 0 ; \quad y_{m}, \gamma_{\lambda} \subset C_{2}\left(Q \backslash x^{\prime}=0\right)$,

$$
\begin{gathered}
z=\left(t, \xi_{v}\right)^{*}, \quad x(t, 0) \equiv \\
\equiv 0\left(i=\overline{1, k_{1}}, j=\overline{1, k_{2}}, m=\overline{1, k}=k_{1}+k_{2}, \lambda=\overline{1, n-k, v, \sigma=\overline{1, n)},}\right.
\end{gathered}
$$

чтобы использовать известные инварианты $y^{\prime}{ }_{i}(q)$ и функции Ляпунова $y^{\prime \prime}{ }_{j}(q)$ для системы (1.1) при $u \equiv 0 . y_{m}(q)$ - непрерывные, неограниченные функции, одновременно исчезающие лишь при $x^{\prime}=0 . E_{+}{ }^{k}-$ область $0 \leqslant y_{m}<\infty \quad(m=1, k), \Gamma-$ область значений $\gamma_{\lambda}(q)$. Тогда $x(z) \subset C$ в области $D=T \times E_{+}{ }^{k} \times \Gamma$ и $x(z) \subset C_{1}$ на $D_{0}=$ $=T \times E_{0}{ }^{k} \times \Gamma$, где $E_{0}{ }^{k}-$ область $E_{+}{ }^{k} \backslash y=0$. Пусть для любых фиксированных $t^{0} \in T, y^{0} \in E_{+}{ }^{k}$ область $\Gamma$ значений $x^{\prime \prime}\left(t^{0}, y^{0}, \gamma\right)$ совпадает с областью $E^{n-l}$ и выполняются неравенства

$W_{11}\left(x^{\prime}\right) \leqslant|y(q)| \leqslant W_{21}\left(x^{\prime}\right), \quad q \in Q\left(W_{\alpha 1}(0)=0, \quad W_{\alpha 1} \subset C\left(E^{l}\right), \quad \alpha, \beta=1,2\right)$,

$$
W_{12}(y) \leqslant\left|x^{\prime}(z)\right| \leqslant W_{22}(y), z \in D\left(W_{\beta 2}(0)=0, W_{\beta 2} \subset C\left(E_{+}^{k}\right)\right),
$$

где $W_{\alpha \beta}-$ положительно определенные бесконечно большие функции: $W_{11} \rightarrow \infty, \quad\left|x^{\prime}\right| \rightarrow \infty, \quad W_{12} \rightarrow \infty, \quad|y| \rightarrow \infty$. Из (1.5) следует, что $x^{\prime} \rightarrow 0$ лишь при $y \rightarrow 0$ и $\left|x^{\prime}\right| \rightarrow \infty$, если $|y| \rightarrow \infty$. В переменных $\xi$ имеем эквивалентную (1.1) систему

$$
\begin{gathered}
\xi=F(z)+A(z) u, \quad z \in D_{0}, \quad u \in \omega, \quad F(t, 0) \equiv 0, \quad F=\left(Y_{i}, Y_{j}, Z_{\lambda}\right)^{*}, \\
Y_{i}=X\left[y_{i}^{\prime}\right]=0, Y_{j}=X\left[y^{\prime \prime}{ }_{j}\right], Z=X\left[\gamma_{\lambda}\right], X[f]=\partial f / \partial t+X_{v} \partial f / \partial x_{v},
\end{gathered}
$$

$A=\left\|a_{v s}(z)\right\|=P M, \quad \operatorname{rank} A=r, \quad A_{1}=\left\|a_{m s}(z)\right\|, \quad Y=\left(Y_{j}\right)^{*}, \quad Z=\left(Z_{\lambda}\right)^{*}$.

Согласно (1.1) и (1.4), $Y_{j}, Z_{\lambda}, a_{v s} \subset C_{1}\left(D_{0}\right)$. Пусть для $\forall \xi_{0} \equiv \xi\left(t_{0}\right) \in D_{0}$ и допустимого $u(t)$ решения (1.6) существуют, единственны и $z$-продолжаемы $\left[{ }^{1}\right]$ в $D_{0}$. Тогда регулятору $u(z)$ системы (1.6), приводящему точки $D^{0}: t \geqslant 0, y \in E_{+}{ }^{k}, 0<|y| \leqslant d^{0}, \gamma \in \Gamma$ на $y=0$, соответствует допустимый регулятор $u_{1}(q) \_u(t, \xi(q))$ системы (1.1), преобразующий точки $H^{0}: t \geqslant 0,0<\left|x^{\prime}\right| \leqslant h^{0}, x^{n} \in E^{n-l}$ на $x^{\prime}=0$. Для данного $d^{0}>0$ возьмем $h^{0}$ из

$$
W_{21}\left(x^{\prime}\right) \leqslant d^{0} \text { при }\left|x^{\prime}\right| \leqslant h^{0} \quad\left(d^{0}=\text { const, } h^{0}=\text { const }>0\right) .
$$

Если $u(z)$ приводит $D_{0}$ на $y=0$, то $u(t, \xi(q))$ дает $x^{\prime}$-стабилизацию в целом движения $x=0$. Для оптимальной $x^{\prime}$-стабилизации достаточно решить соответствующую ей задачу $y$-стабилизации движения $\xi=0$ системы (1.6). Назовем систему (1.6) и $y_{m}, \gamma_{\lambda}$ информативными, если $(1.4),(1.5)$ выполняются. В частности, эти условия справедливы, когда $y^{\prime}{ }_{i}(q)$ и $y^{\prime \prime}{ }_{j}(q)$ - известные для (1.1) инварианты, $y$-автономные переменные и функции Ляпунова при $u \equiv 0$. Критерий оптимальности $x^{\prime}$-стабилизации - минимум интеграла

$$
\begin{gathered}
I=\int_{t_{0}}^{t_{1}} f(t, \xi, u) d t\left(f=f_{0}(z)+f_{1}(z, u), f_{1}(z, 0) \equiv 0,0 \leqslant f_{0}, f_{1} \subset C\left(D \times E^{r}\right)\right), \\
f_{1}\left(z, u_{2}\right)-f_{1}\left(z, u_{1}\right) \geqslant\left(u_{2}-u_{1}\right) \cdot \partial f_{1} / \partial u_{1}\left(\forall u_{2} \in E^{r}, u_{1} \in E_{0}^{r}\right),
\end{gathered}
$$




$$
0<f(z, u), 0 \leqslant f_{0}(z) \subset C_{1}, 0 \leqslant f_{1}(z, u) \subset C_{2}, \quad z \in D_{0}, u \in E_{0}^{r},
$$

где $f_{1}(\cdot, u)$ - выпуклая функция.

2. Рассмотрим в $\xi$ задачу оптимальной по (1.8) $x^{\prime}$-стабилизации движения $x=0$ системы (1.1), для которой (1.6) - информативная. Оптимальный регулятор $u_{1}{ }^{0}(q) \equiv u^{0}(t, \xi(q))$ и область начальных значений (1.7) будем искать методом Ляпунова с помощью производящей функции $S(t, \xi)$. Обозначим

$$
B[S, u]=f_{0}(z)+F[S]+L[S, u], \quad z=\left(t, \xi_{v}\right)^{*} \in D_{0}^{1}, \quad u \in \omega,
$$

$$
F[S] \equiv \theta_{0}+\theta_{\lambda} Z_{\lambda}+\psi_{j}^{\prime \prime} Y_{j}(z), \quad \theta_{0}=\partial S / \partial t, \quad \theta_{\lambda}=\partial S / \partial \gamma_{\lambda}, \quad \psi_{i}^{\prime}=\partial S / \partial y_{i}^{\prime},
$$

$$
\psi_{j}^{\prime \prime}=\partial S / \partial y^{\prime \prime}{ }_{j}, \quad \theta=\left(\theta_{\lambda}\right)^{*},
$$

$$
L[S, u]=L(z, p, u)=f_{1}+a \cdot u, \quad a=A^{*} p, \quad p=\left(\psi_{i}^{\prime}, \psi^{\prime \prime}{ }_{j}, \theta_{\lambda}\right)^{*},
$$

$$
\psi=\left(\psi_{i}^{\prime}, \psi^{\prime \prime}{ }_{j}\right)^{*}\left(i=\overline{1, k_{1}}, j=\overline{\left.1, k_{2}\right)},\right.
$$

$$
D^{1}=t \geqslant 0, \quad y \in E_{+}^{k}, \quad|y| \leqslant d^{1}, \quad \gamma \in \Gamma ; \quad D_{0}^{1}=D^{1} \backslash y=0,
$$

$$
d^{1}=\operatorname{const}\left(\lambda=\overline{1, n-k,} k=k_{1}+k_{2}\right) \text {. }
$$

Здесь и ниже $S \subset C\left(D^{1}\right) \cap C_{1}\left(D_{0}^{1}\right) . S(z)$ - допустимая функция, а непрерывный в $D_{0}^{1}$ регулятор $u^{0}(z, p) \neq 0$ экстремальный, если

$$
S(t, 0, \gamma) \equiv 0, \quad V_{1}(y) \leqslant S(z) \leqslant V_{2}(y), \quad z \in D^{1}, \quad V_{2}(0)=0,
$$

$L\left[S, u^{0}\right] \leqslant L[S, u], \quad B\left[S, u^{0}\right]=0, \quad f\left(z, u^{0}\right) \geqslant W(y), \quad z \in D_{0}^{1}\left(u^{0} \in \omega_{0}, \quad u \in \omega\right)$,

где $V_{1}(y), V_{2}(y)$ - непрерывные, бесконечно большие и определенноположительные в $E_{+}{ }^{h}$ функции. $W(y)$ - определенно-положительная и непрерывная в $D^{1}$ функция. Следуя $\left[{ }^{1}\right]$, находим $d^{0}$ в (1.7). Обозначим $v^{0}=\inf V_{1}(y)$ при $|y|=d_{0}=d^{1}-\varepsilon_{0}, y \in E_{+}{ }^{k}, \varepsilon_{0}=$ const $>0$. Тогда $d^{0}=d_{0}{ }^{1}-\varepsilon_{1}$, где $d_{0}^{1}-$ минимальный модуль корня $V_{2}(y)=v^{0}, \varepsilon_{1}=$ $=$ const $>0$. Из $(2.2)$ на основании теоремы 3.1 [ $\left.^{1}\right]$ и ее модификации $\left[{ }^{2}\right]$ имеем утверждение.

Т е о рем а 1. Если информативная (1.6) имеет допустимую $S(z) u$ экстремальный регулятор $u^{0}(z, p)$ в $D_{0}{ }^{1}$, то для $\forall q_{0}$ в $0<\left|x_{0}^{\prime}\right| \leqslant h^{0}$ функция $u_{1}{ }^{0}(q) \equiv u^{0}[z(q), p(z(q))]$ - оптимальный регулятор.

Здесь управление $u_{1}^{1}(t)=u_{1}^{0}\left[t, x\left(t, x_{0}\right)\right] \subset C\left[t_{0}, t_{1}\right)$ и выполнены условия $S\left(z_{0}\right)=\min I(u), \quad\left|x^{\prime}\left(t, x_{0}\right)\right| \leqslant \sup W_{22}(y), y \in D^{1} ; x^{\prime}\left(t, x_{0}\right) \rightarrow 0$ при $t \rightarrow t_{1}-0$, где $z_{0}=\left(t_{0}, \xi_{0}\right)^{*}, \quad \xi_{0}=\left(\xi_{v}\left(t_{0}\right)\right)^{*}, \quad q_{0}=\left(t_{0}, x_{v_{0}}\right)^{*}, x_{0}=$ $=\left(x_{v}\left(t_{0}\right)\right)^{*}$. Регулятор $u^{0}(z, p)$ дает оптимальную $x^{\prime}$-стабилизацию в целом, если условия (2.2) выполнены на $D_{0}=T \times E_{0}{ }^{k} \times \Gamma$, где $S(z) \subset C_{1}\left(D_{0}\right)$ и $S(z) \subset C(D)$. Пусть в $D_{0}{ }^{1}$ есть $S(z), u^{0}(z, p)$, удовлетворяющие (2.2). Тогда решение задачи сводится к поиску экстремального $u^{0}(z, p)$ и допустимого решения $S(z)$ уравнения $B^{0}[S] \equiv$ $\equiv B\left[S, u^{0}\right]=0$.

Из условий (1.3), (1.8) находим, что множество точек $\left\{u_{1}{ }^{1}\right\}$, где $L(u)$ достигает inf на $\omega$, есть выпуклый компакт. Множество $\left\{u^{0}\right\}$ не пусто, оно состоит из $\left\{u_{1}{ }^{1}\right\}$ без $u=0$. С учетом (1.3), (1.8) необходимо и достаточно, чтобы $u^{0}$ удовлетворяла

$\partial L / \partial u+\mu \partial v / \partial u=0, \mu\left[v(u)-v^{0}\right]=0\left(0<v(u) \leqslant v^{0}, \mu=\mu(z, p) \geqslant 0\right)$. 
Если $\mu=0$, то решения (2.3) стационарные: $u^{0}=u_{0}$. При $\mu>0$ имеем нестационарные точки $u^{0}=u^{1}, v\left(u^{1}\right)=v^{0}$. Из (1.3), (1.8), (2.1), (2.3) выводим

$L(u)-L\left(u^{1}\right) \geqslant \mu\left(u^{1}-u\right) \cdot \nabla v\left(u^{1}\right) \geqslant \mu\left(v^{0}-v(u)\right) \geqslant 0, \quad L\left(u^{1}\right) \leqslant-\mu v^{0}<0$.

В силу (1.3) и (2.4) для $L(u)=L\left(u^{1}\right)$ необходимо, чтобы $u=-u^{1}$, $v\left(u^{1}\right)=v\left(-u^{1}\right)$. Отсюда находим неравенство

$$
\begin{aligned}
& L\left(u^{1}\right)+L\left(-u^{1}\right)=f_{1}\left(u^{1}\right)+f_{1}\left(-u^{1}\right) \leqslant \\
& \leqslant-2 \mu v^{0}<0(\Phi(z, u)=\Phi(\cdot, u)=\Phi(u)),
\end{aligned}
$$

которое несовместимо с $f_{1} \geqslant 0$ в (1.8). Поэтому $L\left(u^{1}\right)<L(u)$ при $u^{1} \neq u \in \omega$. Отсюда для $\forall z \in D_{0}{ }^{1}$ имеем альтернативу: либо система (2.3) имеет единственное решение $u^{1}(z, p)$, либо $\left\{u_{0}\right\}$ не пусто и нестационарного решения (2.3) не существует. Пусть $S(z)$ такая, что $\partial L / \partial u \neq 0$ для всех $z \in D_{0}{ }^{1}, u \in \omega_{0}$. Этот вариант назовем нестационарным режимом. Тогда в $D_{0}^{1}$ имеем единственный нестационарный регулятор $u^{1}(z, p) \subset C_{1}\left(D_{0}^{1}\right)$ и неравенства

$\partial L / \partial u \neq 0, L\left(u^{1}\right)<L(u), v\left(u^{1}\right)=v^{0} \quad\left(z \in D_{0}^{1}, u^{1} \neq u \in \omega, L\left(u^{1}\right)<0\right)$.

Стационарным режимом назовем вариант, когда $\mu=0$ и (2.3) имеет для всех $z \in D_{0}{ }^{1}$ хотя бы одно решение $u_{0}(z, p) \in \omega_{0}, u_{0} \subset C_{1}\left(D_{0}^{1}\right)$. При этом

$\partial L / \partial u_{0}=0, L\left(u_{0}\right) \leqslant L(u), 0<v\left(u_{0}\right) \leqslant v^{0} ; L\left(u_{0}\right) \leqslant 0, u \in \omega\left(z \in D_{0}^{1}\right)$.

Ограничимся рассмотрением двух режимов - эллиптического и конического. В эллиптическом случае выполнены $(1.8)$, а $f_{1}(z, u)$ строго выпуклая по $u$ функция, причем удовлетворяющая неравенству

$$
f_{s \sigma} v_{s} v_{\sigma}>0, f_{s \sigma}=\partial^{2} f_{1} / \partial u_{s} \partial u_{\sigma}\left(z \in D_{0}^{1}, u, v \in E_{\alpha}^{r}, s, \sigma=\overline{1, r}\right)
$$

В коническом, кроме (1.8), удовлетворяются условия

$$
f_{1}>0, \quad u \cdot \partial f_{1} / \partial u=f_{1}, \quad u \in E_{0}^{r}, \quad f_{1}(z, 0) \equiv 0, \quad z \in D_{0}^{1},
$$

$$
f_{s \sigma} v_{s} v_{\sigma}>0, \quad|u||v|>|(u \cdot v)|, \quad \operatorname{det}\left\|f_{\beta \gamma}\right\|>0 \quad(\beta, \gamma=\overline{1, r-1)} .
$$

Здесь $f_{1}(\cdot, u)$ строго выпуклая на нецентральном интервале $\alpha u_{1}+(1-\alpha) u_{2}$.

2.1. Найдем в эллиптическом случае оптимальные регуляторы для стационарного и нестационарного режимов. Рассмотрим стационарный режим. Для решения $u_{0}$ системы (2.6) из (2.7) выводим $L(u)>L\left(u_{0}\right)$, $u_{0} \neq u \in \omega, u_{0} \in \omega_{0}$. Отсюда следует, что в $D_{0}{ }^{1}$ существует единственный стационарный регулятор $u_{0} \subset C_{1}\left(D_{0}^{1}\right)$. Получим его преобразованием Лежандра. Введем для сопряженных переменных, функции и якобиана обозначения

$$
\begin{gathered}
v_{s}=v_{s}(z, p, u)=\partial L / \partial u_{s}, \quad H^{\prime}{ }_{1}(z, p, u)=v \cdot u-L(z, p, u), \\
\Delta_{1}=\operatorname{det}\left\|\partial^{2} L / \partial u_{s} \partial u_{\sigma}\right\|=\operatorname{det}\left\|f_{s \sigma}\right\| \quad(s, \sigma=\overline{1, r}),
\end{gathered}
$$

где $\Delta_{1}>0$ в силу (2.7). Отсюда имеем равенства 


$$
u_{s}=u_{s}(z, p, v)=\partial H_{1} / \partial v_{s}, \quad H_{1}=H_{1}^{\prime}(z, p, u(z, p, v)) .
$$

Подставляя (2.9) в $(2.6),(2.10)$, найдем стационарный $u_{0}$ и равенства $u_{0}(z, p)=\partial H_{1} / \partial v_{0}, v_{0}=0 ; \quad L\left(z, p, u^{0}\right)=-H_{1}(z, p, 0) \equiv-G_{1}(z, p)$. С учетом (2.1), (2.9)-(2.11) уравнение Беллмана имеет вид

$$
B_{1}^{0}[S]=f_{0}(z)+F[S]-G_{1}(z, p)=0, \quad z \in D_{0}^{1} .
$$

При (1.8), (2.7) на основании теоремы 1 для стационарного режима имеем утверждение: если решение (2.12) удовлетворяет условиям

$$
\begin{aligned}
& S_{1}(t, 0, \gamma) \equiv 0, \quad V_{1}(y) \leqslant S_{1}(z) \leqslant V_{2}(y), \\
& F\left[S_{1}\right]+a \cdot \partial H_{1} / \partial v_{0} \leqslant-W(y), \quad z \in D_{0}^{1},
\end{aligned}
$$

то (2.11) - оптимальный по (1.8), (2.7) регулятор $x^{\prime}$-стабилизации в $C^{0}: 0<\left|x_{0}^{\prime}\right| \leqslant h^{0}$. Здесь $z=z(q), p=\partial S_{1} / \partial \xi$ при обозначениях (1.4), (1.6), (1.7), (2.1), (2.2), (2.9)-(2.11).

Пусть (1.6), (1.8) удовлетворяют условиям $y$-автономности

$f_{0}=\tau f_{0}^{0}(y), \quad Y_{j}=\tau Y_{j}^{0}(y),\left.\quad G_{1}\right|_{\Theta=0}=\tau g_{1}(y, \psi), \quad z \in D_{0}^{1}(\tau=\tau(z)>0)$,

$$
f_{0}^{0}, Y_{j}^{0}, \tau \subset C\left(D_{0}^{1}\right), \quad \theta=\left(\partial S / \partial t, \partial S / \partial \gamma_{\lambda}\right)^{*} \quad(\lambda=\overline{1, n-k)},
$$

где $g_{1}, f_{0}{ }^{0}, Y_{j}{ }^{0}$ не зависят от $t, \gamma$. Решение $(2.12)$ ищем в виде $S_{1}{ }^{0}(y)$ :

$$
\begin{gathered}
b_{1}^{0}[S]=\left.\tau^{-1} B_{1}^{0}[S]\right|_{\Theta=0}=f_{0}^{0}(y)+\psi_{j}^{\prime \prime} Y_{j}(y)-g_{1}(y, \psi)=0, \\
y \in E_{+}^{k}, \quad 0<|y| \leqslant d^{1} .
\end{gathered}
$$

(2.12) сводится к (2.15) при условиях $y$-автономности (2.14). Здесь уменьшена размерность задачи, упрощены условия (2.13) оптимальности (2.11). Из (2.13)-(2.15) получаем результат.

Т е о рем а 2. Если выполнены условия (2.14) и определенно-положительная функция $S_{1}{ }^{0}(y)$ в $D_{0}{ }^{1}$ удовлетворяет (2.15) и неравенству

${ }_{\tau} Y_{j}^{0} \psi_{j}^{\prime \prime}+u_{0}^{0} \cdot A_{1}^{*} \psi \leqslant-W(y)\left(u_{0}^{0}=\partial H_{1} /\left.\partial v_{0}\right|_{\Theta=0}, v_{0}=0 ; \psi=\frac{\partial S_{1}^{0}}{\partial y}\right)$,

то вектор-функция (2.11) при $\theta=0$ - оптимальный по (1.8), регулятор х'-стабилизации для $\forall q_{0}$ в $C^{0}$.

При нестационарном режиме зададим преобразование равенствами

$$
\begin{gathered}
v(z, p, \mu, u)=\partial R / \partial u, u(z, p, \mu, v)=\partial H_{2} / \partial v \quad\left(z \in D_{0}^{1}, u \in \omega_{0}\right), \\
R=L+\mu v, \quad H_{2}(z, p, \mu, v)=u \cdot v-R(z, p, \mu, u)\left(u \cdot v=\sum_{s=1}^{r} u_{s} v_{s}\right) .
\end{gathered}
$$

В силу (1.3), (2.7) $\mu>0$, якобиан $\Delta_{2}^{\prime}=\operatorname{det}\left\|\partial^{2} R / \partial u_{s} \partial u_{\sigma}\right\|>0$. Из $(2.3),(2.17)$ находим вид нестационарного экстремального регулятора и уравнение для $\mu$ :

$$
u^{\prime}=u^{\prime}(z, p, \mu)=\partial H_{2} / \partial v_{0}, \quad v\left(\partial H_{2} / \partial v_{0}\right)=v^{0}, \quad v_{0}=0 .
$$


Для $(2.18\}$ якобиан $\Delta_{2}=\left(\partial v / \partial u_{\sigma}^{\prime}\right)\left(\partial u_{\sigma}^{\prime} / \partial \mu\right) \neq 0$ при $z \in D_{0}{ }^{1}$. Дифференцируя по $\mu$ тождества (2.3) при $u:=u^{\prime}(z, p, \mu)$, найдем

$$
\begin{gathered}
r_{\sigma s}^{\prime} \partial u_{s}^{\prime} / \partial \mu=-\partial v / \partial u_{\sigma}^{\prime}, \\
\Delta_{2}=-r_{\sigma s}^{\prime}\left(\partial u_{s}^{\prime} / \partial \mu\right)\left(\partial u_{\sigma}^{\prime} / \partial \mu\right)\left(r_{\sigma s}^{\prime}=\partial^{2} R / \partial u_{\sigma}^{\prime} \partial u_{s}^{\prime}\right) .
\end{gathered}
$$

Тогда из $u^{\prime} \cdot \partial v / \partial u^{\prime}=v^{0} \neq 0, \quad r_{s \sigma} u_{s} u_{\sigma}>0$ имеем $\Delta_{2}<0$. Подставляя решение $\mu=M_{1}(z, p)$ этого уравнения в (2.18), получим экстремальный регулятор $u_{2}{ }^{1}=u^{\prime}\left(z, p, M_{1}(z, p)\right) \subset C_{1}\left(D_{1}^{0}\right)$. Далее находим

$$
\partial H_{2} / \partial \mu=-\partial R / \partial \mu=-v(u), \quad G_{2}(z, p) \equiv v^{0} M_{1}+H_{2}\left(z, p, M_{1}, 0\right) .
$$

Из (2.17)-(2.19) для нестационарного режима получаем уравнение

$$
B_{2}^{0}[S]=f_{0}(z)+F[S]-G_{2}(z, p)=0, \quad z \in D_{0}^{1}\left(L\left(z, p, u_{2}^{1}\right)=-G_{2}(z, p)\right) .
$$

Условия $y$-автономности и вид уравнения Беллмана получаем из (2.14) и $(2.15)$ заменой $G_{1}, g_{1}$ на $G_{2}, g_{2}$. Аналогично из $(2.13)$ и $(2.16)$ находим условия оптимальности $u_{2}{ }^{1}$, произведенного $S_{2}(z)$ или $S_{2}{ }^{0}(y)$.

2.2. Найдем оптимальный регулятор в коническом случае (1.8), (2.8). Рассмотрим стационарный режим, где по условию существует хотя бы один стационарный регулятор $u_{0}$, удовлетворяющий (2.6). Из (2.6) с учетом (1.3), (1.8), (2.8) выводим

$L\left(u_{0}\right)=u_{0} \cdot \nabla L\left(u_{0}\right)=\delta^{-1}\left|u_{0}\right|\left|u_{0}^{\prime}\right|^{-1} L\left(\delta u_{0}^{\prime}\right)=0\left(0<\delta \leqslant 1, u_{0}^{\prime}=u_{0} v^{0} v^{-1}\left(u_{0}\right)\right)$.

В силу (2.20) множество $\left\{u_{0}\right\}$ содержит луч $\delta u_{0}^{\prime}\left(v\left(u_{0}^{\prime}\right)=v^{0}\right)$. Из $(2.8)$ следует, что луч $\delta u_{0}^{\prime}$ содержит все множество $\left\{u_{0}\right\}$. Модифицируя преобразование Лежандра, найдем граничный стационарный регулятор. Обозначим

$$
v_{s}=v_{s}(z, p, e)=\partial L / \partial e_{s}, e_{s}=|u|^{-1} u_{s}\left(\partial L / \partial e_{s}=\partial L / \partial u_{s},|e|=1\right) .
$$

Дифференцируя $(2.8)$ по $u_{s}$, получим систему с $\left|f_{s \sigma}\right|=0$ на $D_{0}^{1} \times E_{0}^{r}$

$$
f_{s \sigma} e_{s}=0\left(f_{s \sigma}=\partial^{2} f_{1} / \partial u_{s} \partial u_{\sigma}, u \in E_{0}^{r} ; s, \sigma=\overline{1, r}\right),
$$

где $v_{s}(z, p, e), z, p$ функционально зависимы. Для определения $e_{s}(z, p, v)$ возьмем систему

$$
\partial L / \partial e_{\gamma}=v_{\gamma}, \quad e \cdot e=1 \quad(\gamma=\overline{1, r-1}) .
$$

В силу $(2.8),(2.22)$ ее якобнан не равен нулю. Используя зависимость $v_{s}, z, p$, представим решение $(2.21)$ в симметричном виде $e_{s}=\varrho_{s}(z, p, v)$. Используя $(2.8),(2.21)$ и функцию

$$
H_{3}(z, p, v)=v \cdot \varrho(z, p, v)-L(z, p, \varrho(z, p, v)), \varrho=\left(\varrho_{s}\right)^{*},
$$

найдем $\mathrm{Q}_{s}(z, p, v)$ и связь на $v_{s}$

$$
e_{s}=\varrho_{s}(z, p, v)=\partial H_{3} / \partial v_{s}, \quad H_{3}(z, p, v)=0 .
$$

Функция $H_{3}(z, p, v)$ определяется равенством $H_{3}=\sup (v \cdot e-L)$ при $|e|=1$. Подстановкой $(2.21)$ в $(2.6)$ из (1.3), (2.24), $(2.1)$ находим

$$
u_{0}^{\prime}=v^{0} v^{-1}\left(\partial H_{3} / \partial v_{0}\right) \partial H_{3} / \partial v_{0}, u_{0}=\delta u_{0}^{\prime}, v_{0}=0,0<\delta \leqslant 1 .
$$

Для $S$ имеем систему из $(2.20)$ и линейного уравнения 


$$
\begin{gathered}
B_{3}^{(2)}[S]=G_{3}(z, p)=0, \\
B_{3}^{(1)}[S]=f_{0}(z)+F[S]=0\left(G_{3}=\left.H_{3}\right|_{v_{0}=0}=-L\left(z, p, u_{0}^{\prime}\right)\right) .
\end{gathered}
$$

При достаточно гладких $f_{0}, Y_{j}, Z_{\lambda}, G_{3}$ функция $S_{3}(z)$ должна удовлетворять полной системе $B_{3}{ }^{(\alpha)}[S]=0\left(\alpha=\overline{1, \alpha^{0}} \leqslant n+1\right)$, которую производит из (2.26) скобка Пуассона. Если рассматриваемый случай $y$-автономный

$f_{0}=\tau f_{0}^{0}(y), Y_{j}=\tau Y_{j}^{0}(y),\left.G_{3}\right|_{\Theta=0}=\tau g_{3}(y, \psi), \tau=\tau(z)>0, z \in D_{0}^{1}$,

то соответствующая ему полная система $y$-автономна

$$
\begin{gathered}
b_{3}^{(1)}[S]=f_{0}^{0}(y)+\psi_{j}^{\prime \prime} Y_{j}(y)=0, \\
b_{3}^{(2)}[S]=g_{3}(y, \psi)=0, b_{3}^{(\beta)}[S]=0\left(\beta=\overrightarrow{3, \beta^{0}} \leqslant k\right) .
\end{gathered}
$$

Условия оптимальности регуляторов $(2.25)$ для $B_{3}^{(\alpha)}[S]=0$ имеют вид $S_{3}(t, 0, \gamma) \equiv 0, \quad V_{1}(y) \leqslant S_{3}(z) \leqslant V_{2}(y), \quad F\left[S_{3}\right] \mp \delta a \cdot u_{0}^{\prime} \leqslant-W(y)^{\prime}, \quad z \in D_{0}^{\frac{1}{2}}$,

$$
u_{0}^{\prime}=v^{0} v^{-1}\left(\partial H_{3} / \partial v_{0}\right) \partial H_{3} / \partial v_{0}, v_{0}=0 ; \quad 0<\delta \leqslant 1(p=\partial S / \partial \xi) .
$$

В силу $f_{1}\left(\cdot, u_{0}^{\prime}\right)>0, L\left(\cdot, u_{0}^{\prime}\right)=0$ имеем $\left(a \cdot u_{0}^{\prime}\right)<0$. Отсюда следует, что регулятор $\delta u_{0}^{\prime}$ будет оптимальным, если существуют $\delta, S_{3} \subset C_{1}\left(D_{0}^{1}\right)$, удовлетворяющие (2.29). В случае (2.27) утверждение об оптимальности (2.25) при $\theta=0$ для решения $S_{3}{ }^{0}(y)$ системы (2.28) аналогично теореме 2 . Здесь следует заменить $(\theta=0) S_{1}{ }^{0}, H_{1}$ и $u_{0}^{0}$ на $S_{3}{ }^{0}, H_{3}$ и $u_{0}^{\prime}$ в условиях (2.14), (2.16)

Найдем единственный экстремальный регулятор $u_{4}{ }^{0}$ в нестационарном подслучае (1.8), (2.8). Используя переменные

$$
\begin{gathered}
v_{s}=\partial R / \partial e_{s}, \quad R=L(z, p, u)+\mu v(u)\left(e=|u|^{-1} u, R_{1}=\left.R\right|_{u=e}\right), \\
H_{4}(z, p, \mu, v)=\sup _{e}[v \cdot e-R(z, p, \mu, e)](|e|=1),
\end{gathered}
$$

получим $e_{s}=e_{s}(z, p, \mu, v)=\partial H_{4} / \partial v_{s}$. Из (1.3), (2.3), (2.8), (2.30) находим

$$
u_{4}^{0}\left(z, p, M_{2}\right)=v^{0} v^{-1}\left(\partial H_{4} / \partial v_{0}\right) \partial H_{4} / \partial v_{0}, \quad v_{0}=0 .
$$

$M_{2}=M_{2}(z, p)$ обозначает решение уравнения $H_{4}(z, p, \mu, 0)=0$. Предполагается, что в $D_{0}^{1}$ оно существует и единственно. Подставляя (2.31) в $(2.30)$, получим $L\left(z, p, u_{4}{ }^{0}\right)=-v^{0} M_{2}$ и вид уравнения Беллмана для нестационарного режима

$B_{4}^{0}[S]=f_{0}(z)+F[S]-G_{4}(z, p)=0, \quad z \in D_{0}^{1}\left(G_{4} \equiv v^{0} M_{2}(z, p)\right)$.

Условия оптимальности регулятора $(2.31)$ для решения $S_{4}(z)$ уравнения (2.32) находим из (2.29) заменой $S_{3}, H_{3}, u_{0}$ при $\delta \equiv 1$ на $S_{4}, H_{4}, u_{4}{ }^{0}$. В силу $B^{0} \subset \operatorname{conv}(p)$ можно искать $S$ в виде выпуклой функции от $\xi$.

3. Пр и м ер. Проведем субоптимальную $x^{\prime}$-стабилизацию в $\xi(t, x, \alpha)$ переменных агрегации по функционалу $\left[{ }^{3}\right]$, используя псевдоавтономность $\left[{ }^{3}\right]$ и внутреннюю аппроксимацию $\omega^{\prime}$ для $\omega^{0}=\left\{|u| \leqslant \varrho_{0}\right\}$ при $\operatorname{dim} y \leqslant \operatorname{dim} u$. Гамильтониан, функционал и $\omega^{\prime}$ задачи имеют $\left[^{3}\right]$ значения 


$$
\begin{gathered}
H^{\prime}[S] \equiv H\left[S^{\prime}\right]+h^{\prime}= \\
=p_{0}+p \cdot Y_{1}^{0}(t, y, \alpha)+f_{1}^{0}(t, y, \alpha)+K\left(z, \alpha, v^{\prime}, \gamma\right), \quad p=\partial S / \partial y, \\
-h^{\prime} \equiv f_{2}^{0}(z, \alpha)+f_{2}^{1}(z, u, \alpha)+p \cdot\left[Y_{2}(z, \alpha)+Y^{(2)}(z, u, \alpha)\right], \\
K=f_{1}^{1}\left(t, y, v^{\prime}, \alpha\right)+v^{\prime}(b \cdot \gamma), \\
I^{\prime}=\int_{t_{0}}^{t_{1}}\left(f_{0}+h^{\prime}\right) d \tau,\left.f_{1}^{1}\right|_{v^{\prime}=0} \equiv 0, \omega^{\prime}=\left\{u \mid v^{\prime} \leqslant v_{0}\right\}, \\
v^{\prime}(u, q)=v^{\prime}(-u, q)=\left.v\left(C^{*} u, \alpha\right)\right|_{\alpha=0}, \\
b=A_{1}^{*} p+b^{1} \neq 0, u=v^{\prime} \gamma, C=\left\|a_{\sigma k}^{1^{*}}, d_{\sigma h}\right\|, \\
d_{\sigma h}=d_{\sigma h}(q, \alpha)(\sigma=\overline{1, r}, k=\overline{1, l,} h=\overline{1, r-l}),
\end{gathered}
$$

где $\operatorname{det} C \neq 0, \operatorname{rank} A_{1}=\operatorname{dim} y=l, \partial f_{1}{ }^{1} / \partial v^{\prime}$ монотонна по $v^{\prime}$. Функции $v, d_{\sigma h}$ выбраны из условия максимума объема $\omega^{\prime}$ по $C^{0}$-мере и неравенств

$v_{0} \leqslant \varrho_{0} \lambda_{0} \min _{e} v, \lambda_{0}^{2}=\min \lambda^{2} \geqslant \lambda^{0}(\alpha)>0\left(\left|u^{\prime}\right| \leqslant \varrho_{0},\left|C C^{*}-\lambda^{2} E\right|=0\right)$,

\section{$v_{0} \geqslant \varrho^{0} \lambda_{*} \max _{e} v, \lambda_{*}^{2}=\max \lambda^{2} \leqslant$}

$$
\leqslant \lambda^{*}(\alpha)<\infty\left(\left|u^{\prime}\right| \geqslant \varrho^{0}>0,\left\{u^{\prime}\right\}=F r \omega^{\prime}, v=v(e, \alpha),|e|=1\right),
$$

$C^{-1} b^{1} \equiv b_{1}(t, y, \alpha)$, достаточных для $\varrho^{0} \leqslant\left|u^{\prime}\right| \leqslant \varrho_{0}$ и $t, y$-автономности $S^{\prime}=S^{\prime}(t, y, \alpha)$. При линейной, вогнутой и выпуклой $f_{1}{ }^{1}\left(v^{\prime}, \cdot\right), d>0$ (в нестационарном режиме) из (3.1), (3.2) находим экстремальные регуляторы и уравнение Беллмана

$u_{\tau}^{0}=0, \quad h<0, \quad B_{\tau}^{0}\left[S^{\prime}\right]=L\left[S^{\prime}\right] \equiv p_{0}+p \cdot Y_{1}(t, y, \alpha)+f_{1}^{0}(t, y, \alpha)=0(\tau=\overline{1,3})$,

$$
u_{\tau}^{0}=v_{0} \gamma^{0}, h>0, B_{\tau}^{0}\left[S^{\prime}\right]=L\left[S^{\prime}\right]+f_{1}^{1}\left(t, y, v_{0}, \alpha\right)-v_{0} w_{0}(t, y, p, \alpha)=0 \text {, }
$$

$$
u_{i}^{0}=\beta_{i} v_{0} \gamma^{0}, \quad h=0,
$$

$$
B_{i}^{0}\left[S^{\prime}\right]=L\left[S^{\prime}\right]=0\left(0 \leqslant \beta_{1} \leqslant 1, \beta_{2}=0,1, p_{0}=\partial S^{\prime} / \partial t, i=1,2\right) \text {, }
$$

$$
h=1-f_{1}^{1}\left(v_{0}, \cdot\right) / v_{0} w_{0},
$$

$$
w_{0}=w^{\prime}(b, z, \alpha)=w\left(\theta+b_{1}, \alpha\right)\left(\theta=\left(p_{k}, 0_{h}\right)^{*}, h=\overline{1, r-l}\right),
$$

где

$$
w^{\prime}(v, \cdot)=\max _{\gamma}(v \cdot \gamma)=
$$

$$
=w(u, \alpha) \in\{v(u)\}\left(v^{\prime}(\gamma, \cdot)=1, u \equiv C^{-1} v, w(\lambda u)=\lambda w(u)\right),
$$

$-\gamma^{0}=\partial w^{\prime} / \partial b=C^{-1^{*}} \partial w / \partial u_{0}, \quad u_{0}=C^{-1} b, \quad d \equiv \partial K(0) / \partial v^{\prime} \cdot \partial K\left(v_{0}\right) / \partial v^{\prime}$.

При $d \leqslant 0$ для выпуклой $f_{1}{ }^{1}\left(v^{\prime}, \cdot\right)$ имеем стационарный режим, в котоpom

$$
\begin{gathered}
u_{4}^{0}=\left[(1-\beta) v_{1}+\beta v_{2}\right] \gamma^{0}=\left(\partial P / \partial g_{0}\right) \cdot \gamma^{0}, \\
B_{4}^{0}\left[S^{\prime}\right]=L\left[S^{\prime}\right]-P\left(g_{0}, \cdot\right)=0(0 \leqslant \beta \leqslant 1),
\end{gathered}
$$

$v_{1}=\min v_{*}^{\prime}, \quad v_{2}=\max v_{*:}^{\prime} \quad \partial f_{1}^{1} / \partial v_{*}^{\prime}=w_{0} ; \quad P(g, t, y, \alpha)=$ 


$$
=g \cdot v^{\prime}-f_{1}^{1} \geqslant 0\left(g_{0}=w_{0}\right),
$$

$g=\partial f_{1}^{1} / \partial v^{\prime}, \quad v^{\prime}=\partial P / \partial g, \quad \partial^{2} P / \partial g^{2}>0 ; \quad P\left(g_{0}\right)>0, \quad 0 \equiv\left\{v_{*}^{\prime}\right\}\left(v_{2}-v_{1}<v_{0}\right)$.

Уравнения (3.3), (3.4) имеют размерность $l \ll n$. Пусть $u_{\tau}{ }^{0}, u_{4}{ }^{0}$ дают $x^{\prime}$-стабилизацию $(1.1),(3.1)$ при условиях теоремы 1 или 2. Тогда $u_{\tau}{ }^{0}, u_{4}{ }^{0}$ - субоптимальные регуляторы системы (1.1), (3.1). Минимизация по вектору агрегации $\alpha$ проводится аналогично $\left[{ }^{3}\right]$.

\section{ЛИТ Е РА Т У РА}

1. Р у м ян це в В. В., Прикл. мат. и мех., 34, вып. 3, 440-456 (1970).

2. К ей с И., Изв. АН ЭССР, Физ. Матем., 24, № 2, 178-187 (1975).

3. К е й с И., Изв. АН ЭССР, Физ. Матем., 29, № 1, 94-96 (1980).

Институт кибернетики

Академии наук Эстонской ССР
Поступила в редакцию 13/VII 1979

\section{KEIS}

\section{INFORMATIIVSETE MUUTUJATEGA SUSTEEMIDE OPTIMAALNE JUHTIMINE}

Artiklis on käsitletud dünaamiliste süsteemide liikumise optimaalset stabiliseerimist osa (või kõigi) muutujate suhtes. Vastava kriteeriumi funktsioon on kumeras kompaktses piirkonnas asuvate juhtivjōudude järgi kumer. On esitatud Bellman-Jacobi võrrandi kujud erinevate ekstremaalsete režiimide puhuks ning tõestatud teoreemid globaalse ja lokaalse optimaalse juhitavuse tingimuste kohta.

\section{KEIS}

\section{AN OPTIMAL CONTROL SYNTHESIS FOR THE SYSTEM WITH INFORMATIVE VARIABLES}

The optimal $x^{\prime}$-stabilization problem for large-scale system is considered. The Hamiltonian is a supposed convex function of controls lying in convex-compact domain of constraints. The state vector $x$ is $n$-dimensional, its component $x^{\prime}$ being an $m$-dimensional vector. New aggregation variables composed of observable and $x^{\prime}$-stable components $y_{k}(k \ll n)$, are introduced in the form of a Lyapunov vector function. Sufficient conditions for optimal contrallability are established in Theorems 1, 2. They represent a modification of the Krasovskii-Rumyantsev's results. The classification covering various extremal control policies, is also provided. The suboptimal control policy is derived by applying preceding results to a class of $y$-autonomous systems. 